\title{
Ralf Oberndörfer
}

\section{Studentische Politik oder politische StudentInnen}

\section{Der umworbene akademische Nachwuchs auf der Suche nach neuen Aktionsformen}

StudentInnen sind in. Mit Umfragen traktiert und von verschiedenen gesellschaftlichen Blickwinkeln aus begutachtet sind sie spätestens seit dem Herbst 1995 fester Bestandteil in der Debatte über den Standort Deutschland. Sie sollen die ProtagonistInnen einer hoffentlich ganz anderen Politik sein. Nicht wenige wünschen, sie würden endlich politisch agieren, nicht immer nur abwarten. Hatte nicht auch Ende der sechziger Jahre eine StudentInnengeneration einen längst überfälligen Modernisierungsschub bewirkt, der zum Bau des »Modell Deutschland « notwendig war? Hatte eine rebellische Studierendengeneration nicht geholfen, endlich die totgeschwiegene Nazi-Vergangenheit öffentlich zu machen und zu bewältigen, die Wirtschaftswunder-Gesellschaft zu zivilisieren?

Stärker als je zuvor in den letzten fünfzehn Jahren werden 1996 die Krise der Universität und mögliche Alternativkonzepte $\mathrm{zu}$ ihr hochstilisiert zu einer Identitätsfrage des neuen Deutschland, das im sechsten Jahr nach der Wiedervereinigung nicht mehr weiter weiß. Es ist nichts Neues, daß staatliche Sinnsuche sich verbindet mit einer Diskussion um die Reorganisation von Wissenschaft. Wichtigstes Beispiel ist die Gründung der Berliner Universität im Jahr 1809. Die Reformer des im Jahre 1806 vernichtend geschlagenen Preußen sahen die Altertümlichkeit ihres Staates und verpaßten ihm kurzerhand eine neue Wissenschaftsstruktur. Daß die 1505 gegründete Universität in Frankfurt/Oder 1811 kurzerhand nach Breslau verlegt wurde, weil sie Berlin Konkurrenz machte, ist dabei so richtig nach dem Geschmack der Umstrukturierer von heute, die am liebsten ganze Hochschulen verschwinden lassen würden. Das damals für den Standort Preußen entwickelte Humboldtsche Konzept der Einheit von Lehre und Forschung an einer säkularen Hochschule hatte in der Tat bahnbrechende Folgen. Bis auf den heutigen Tag wird es, beispielsweise in Die Zeit, flehentlich beschworen: »Humboldts Erbe wird verspielt« (Fritz-Vannahme 1996, 1) und »Wenn das Humboldt wüßte...« (Zimmer 1996, 35) sind typische Überschriften für einen Diskurs, der die deutsche Universität gerne wieder an alte Größe anknüpfen sehen möchte. Allein, kein neuer Humboldt ist in Sicht und Preußen gibt es auch nicht mehr. 
Nicht nur daher kommt das liebevolle Interesse, das StudentInnen neuerdings mehrheitlich entgegengebracht wird. Von allen Seiten wird ihnen angetragen, sich gefälligst politisch zu beteiligen, Mitverantwortung zu übernehmen für die Uni und die Gesellschaft von morgen, während Politisierung ja nach 1968 etwas Anrüchiges war. Der Präsident der FU äußert offen Sympathie für studentische Proteste. Der Chefredakteur der im Auftrag des Präsidenten erscheinenden FU-Nachrichten (FU:N) schreibt:

»Nicht nur die Studierenden sehen in ihren Aktionen die vielleicht einzige Chance, die Politik zur Vernunft, zu einer Sparpolitik mit Sinn, Verstand und Augenmaß zu bewegen. Deshalb ist ein Ende der Aktionen weder abzusehen, noch aus Sicht der Hochschulen wünschenswert.« (Walther 1996, 2)

Auf den Meinungsseiten der Tageszeitungen räsonnieren JournalistInnen über die Gefahren einer Entpolitisierung der StudentInnen. Anders als vor dreißig Jahren, als die alte Bipolarität von Philister-Student unter den Namen Spießer-Halbstarker noch einmal zu neuem Glanz erblühte, hat die Gesellschaft »Kohls Kinder« (Fritz-Vannahme 1995a, 41 ) ganz fest in ihr Herz geschlossen. Die Jugend von heute soll es nicht einmal besser haben, sie soll es besser machen.

Im folgenden sollen zunächst die vier Erwartungshaltungen kurz skizziert werden, mit denen sich StudentInnen konfrontiert sehen. Danach geht es um die Frage, wie die StudentInnen mit dem Erfolgsdruck umgehen, der auf ihnen lastet. Wo verorten sie sich in der gegenwärtigen Situation? Warum nehmen sie die Zuweisung, »StudentInnen « und sonst gar nichts zu sein, so bereitwillig an und warum macht gerade das ihre Proteste so harmlos? Zuletzt sollen zwei Ansätze vorgestellt werden, die die beklagte Entpolitisierung eventuell überwinden können, wenn auch nicht im Sinne der skizzierten Diskurse.

\section{Radikale Linke und die ewig blöden StudentInnen}

Die meisten VertreterInnen der (radikalen) Linken mögen die StudentInnen nicht und erwarten nichts von ihnen. Jeden Streik, jede Institutsbesetzung, jede Aufmüpfigkeit nehmen sie zum Anlaß, darauf hinzuweisen, daß das ja wohl nicht zur Revolution führen könne. StudentInnen sind einfach unfähig. Im neuesten unikonkret (Sommersemester 1996) ereifert sich konkretHerausgeber Gremliza mal wieder über die 68er im besonderen und die StudentInnen allgemein. Nachdem er Antje Vollmer, die frustrierte sexuelle Revolution und die falsche Auseinandersetzung mit dem Nationalsozialismus in bekannter Weise durchgenudelt hat, stellt er fest:

"Studentenrevolution verhält sich zu Revolution wie Peanuts zu Kapital. Und das Werk der Bürgerkinder kann nur die Befreiung der Bürgerkinder selber sein.« (Gremliza 1996, 2)

Solchermaßen eingestimmt auf den Makel, StudentIn zu sein, darf die Zielgruppe dann Erlebnisaufsätze echter ehemaliger Studenten lesen. Knud 
Kohr, Stefan Ripplinger und Carsten Otte, schreibenderweise bei der überregionalen Heimatzeitung junge Welt beschäftigt, gehen gleich in die vollen. Kohr erzählt bei einem Besuch an Otto-Suhr Institut der FU Berlin sich selbst, was er über StudentInnen weiß: »Die sind gar nicht so dumm. Die sind nur genauso dumm wie du damals« (Kohr 1996, 2). Stefan Ripplinger analysiert luzide: »Studenten sind unfähig zu Protest, denn sonst wären sie keine Studenten. Sie haben es zuvor schon versäumt, den Kindergarten zu schmeißen... « (Ripplinger 1996, 5). Carsten Otte meint, bei Studierenden einen unbändigen Schreibdrang feststellen zu können und fragt: »Was soll denn aus all den schreibbesessenen Hochschulabsolventen werden? « (Otte 1996, 23). Kein Problem, Konkret und junge Welt haben ganz viel Platz für sie. Und wenn sie das nicht auslastet, fallen sie in eigens dafür angefertigten Heftchen über »die « StudentInnen her, weil's Laune macht und Konsens schafft wie sonst nur ein Herrenwitz am Stammtisch. »Vergeßt 68! «, so lautet der Titel von Gremlizas Leitartikel. Die StudentInnen heute sollen vergessen, daß 68 nicht nur Antje Vollmer und Daniel Cohn-Bendit, sondern auch Gudrun Ensslin und Peter Brückner bedeutete, daß es diese Generation war, die die Texte des Instituts für Sozialforschung und damit die »Kritische Theorie« wiederentdeckte, daß 1959 5,2\% Studierende aus Arbeiterfamilien kamen und 1985 17\% (Mahlmann 1994, 56), um nur einige wichtige Kleinigkeiten zu nennen. Die Botschaft ist simpel: Die StudentInnen von heute sind so doof wie die Studenten von damals, nichts darf übrigbleiben als politische Perspektive. Das einzige, was Rettung verspricht, ist ein Konkret-Abo. Wer studiert und Konkret nicht abonniert, wird enden wie Kohl, lernt man auf Seite 13. Denn der kannte Konkret nicht, bestimmt genauso wenig wie Cohn-Bendit und Vollmer und all die anderen schrecklichen 68er jemals Konkret zu ihrer Rettung gelesen haben. Denn hätten sie nur einmal bis 1973 einen Blick in Röhls SoftPorno-Postille geworfen, dann müßten sie bestimmt nicht als Bundestagsvizepräsidentinnen mit Sudetendeutschen reden, sondern dürften StudentInnen im Jahr 1996 vorlügen, daß wahre Politisierung bei der Wahl der richtigen Zeitschrift beginnt.

Soviel zu diesen radikalen Linken. Ihr StudentInnenbild ist hausbacken, starr gefügt, veraltet. In den übrigen Segmenten des Diskurses über StudentInnen, bei Liberalen, Grün-Alternativen und Konservativen, geht es wesentlich subtiler zu.

\section{Liberale und die pragmatischen Studentlinnen}

Im Oktober 1995 veröffentlichte Die Zeit die Ergebnisse einer Studie Student '95, die Infratest Burke Berlin aufgrund einer Befragung von 1019 StudentInnen im Sommer 1995 durchgeführt hatte (Student '95 1995a, 43; 
Student '95 1995b, 47). Dieser Umfrage zufolge halten sich 68\% der StudentInnen für angepaßt, 77\% für karrierebewußt und 39\% für unkritisch (Student '95 1995b, 47). Drei Viertel glauben, daß Deutschland auf dem Weltmarkt eine gute Figur macht und $42 \%$ sind sich sicher, daß diese Position Deutschlands in den nächsten zehn Jahren noch gestärkt werden wird (ebd.). Trotzdem oder gerade deswegen lobt der Autor des begleitenden Kommentars diese Generation in den höchsten Tönen (Fritz-Vannahme 1995a, 1995b). Anders als die StudentInnen in einer ähnlichen Studie 1979, suche die 95er-Generation »nicht mehr die Veränderung des Systems, sondern dessen Verbesserung « (Fritz-Vannahme 1995a, 42). Bei der Parteiensympathie liegen die Grünen mit $35 \%$ weit vor CDU $(19 \%)$ und SPD (18\%). Die Ökopartei sei es, die diese Generation in das Parteiengefüge der BRD einbinde. Kein Wunder, daß Umweltschutz mit $82 \%$ das wichtigste gesellschaftspolitische Thema ist (Student '95 1995a, 42). Was Fritz-Vannahme (1995a, 43) offenbar so begeistert, ist die Mischung aus edlen Motiven und stillem Pragmatismus, ganz anders als bei den aufgeregten $68 \mathrm{em}$ und den No-Future-Kids von 1979. $\gg$ Die Welt ist so, wie sie ist. Nur darf sie so nicht bleiben «, dies sei das Credo der »empörten Greenpeace-Generation«, die lieber im kleinen Rahmen, in Bürgerinitiativen und privaten Gesprächen die Welt verändern will anstatt in Parteien, nicht zuletzt weil die Hälfte überzeugt ist, daß Politiker »in erster Linie in die eigene Tasche wirtschaften« (ebd., 42). Obwohl Hochschul- und Studienreform nicht mehr die Wichtigkeit genießen wie vor 16 Jahren und die Uni in erster Linie als »Institution zum Erwerb von spezialisiertem Fachwissen« und erst an elfter und letzter Stelle als »Institution, die die Möglichkeit bietet, sich hochschulpolitisch zu betätigen« (Student '95 1995b, 48) gesehen wird, würden sich trotzdem $84 \%$ an hochschulpolitischen Aktionen beteiligen (Student '95 1995a, 43). Spätestens hier stutzt die geneigte LeserIn. Die mickrige Beteiligung an den überregionalen Protestaktionen im Herbst 1995 und die äußerst schleppende Mobilisierung zu den studentischen Protestaktionen in Berlin lassen Zweifel aufkommen. Sind voll verzinstes BAföG und vernichtende Kürzungen mit dem Ziel, die Massenunis kleinzukriegen, einfach nicht die richtigen Themen für Kohls Kinder?

Was irritiert ist, daß das Ergebnis der Umfrage, das zumindest ambivalent ist, mit aller Gewalt schön geschrieben wird. Die Umfrage stellt fest, daß ein Viertel der Studierenden in einer autoritären Gedankenwelt zu Hause ist. $33 \%$ halten die Aussage $»$ Die Ausländer, die bei uns leben, müssen sich unseren Sitten und Gebräuchen anpassen«, für richtig (Student '95 1995a, 42). Und jene 73\%, die sehr starkes Interesse für »fremde Länder und Kulturen haben « (Student '95 1995b, 49), was als Aufgeschlossenheit (Fritz-Vannahme 1995b, 48) gedeutet wird, stehen den 33\% nicht etwa gegenüber, sondern beide Aussagen können die Kehrseiten des gleichen 
Rassismus sein. Einerseits bejahen $47 \%$ die Aussage $» I c h$ kann mir kein Land vorstellen, in dem es mehr Freiheit gibt als in der Bundesrepublik" (Student '95 1995a, 42), andererseits haben $73 \%$ großes Interesse am Thema Rechtsradikalismus/Ausländerfeindlichkeit (ebd.). Ob das politische System, von dem 67\% überzeugt sind (ebd., 41), eine Ursache dafür sein könnte? Auch beim konstatierten Optimismus beschleichen einen Zweifel. 46\% sagen $\gg I c h$ sehe keine ernsthafte Bedrohung für unsere $\mathrm{Zu}-$ kunft, wir werden mit unseren Problemen schon fertig.«, (ebd., 42). Dabei ist ungeklärt, wer mit »wir « gemeint ist - wir StudentInnen, wir Deutsche, wir, die Menschheit? Es bleibt außerdem offen, inwieweit der Optimismus abhängig ist von der sozialen Situation der Befragten. Daß Leute aus Mittelschichten mit absehbaren Aufstiegsmöglichkeiten gerne von der Zukunft reden, ist ja nun wahrlich nichts neues. Ist der Glaube an die eigenen Möglichkeiten vielleicht klassenspezifisch? Wir erfahren es nicht, denn die Antworten sind nicht aufgeschlüsselt nach sozialer Herkunft; daß Ostler ärmer sind als Westler, steht noch irgendwo. Und was passiert, wenn sich die Hoffnung auf ein ökonomisch aufstrebendes Deutschland und die eigenen Karrierepläne nicht erfüllen, wenn sich der erstrebte Durchschnittsverdienst von $6700 \mathrm{DM}$ (Fritz-Vannahme 1995b, 48) nicht einstellt? »Alle setzen ein großes, fast abenteuerliches Vertrauen in die wirtschaftliche Stärke der Bundesrepublik«, ist an anderer Stelle zu lesen (Grunenberg 1995, 1). Aber: »Ihre revolutionären Eltern, die 68er, haben waschechte Pragmatiker aufgezogen « (ebd.). Abenteuerliche Pragmatiker, und dann?' Zurück bleibt der Eindruck, da habe jemand eine Generation von HoffnungsträgerInnen mit Gewalt herbeischreiben bzw. -interpretieren wollen. Und das verwundert nicht. Denn je schwieriger die ökonomische und soziale Situation in Deutschland wird, desto dringlicher der Wunsch der Generation, deren heimliche Sehnsucht die Wiedervereinigung war, den Stab weiterzureichen. Die zivilgesellschaftliche Variante vom Ruf nach dem starken Mann ist die Suche nach vielen qualifizierten, kühlen ManagerInnen, die - weder überheblich noch devot - das große Deutschland auf seinen Platz in der Welt führen werden, ohne dabei die Menschlichkeit zu vergessen. Ob das eine Generation leisten kann, die zur Hälfte Politik, wie vor hundert Jahren große Teile des Bürgertums, für ein schmutziges Geschäft hält?

Ein gutes halbes Jahr später ist der gleiche Autor unzufrieden mit seinen StudentInnen '96, sowohl was Qualität und Quantität ihres Engagements

1 Einen guten Überblick über die Anschlußfähigkeit studentischer Alltagsvorstellungen an (neu)rechte Theoreme bietet Bojadzijev $(1994,3)$, die verschiedene Studien zur politischen Präferenz von Studierenden der letzten Jahre vergleicht. Sie geht auch der Frage nach, weshalb sich StudentInnen häufig links im politischen Spektrum verorten, aber gleichzeitig rechte Positionen vertreten. 
betrifft. $\mathrm{Zu}$ den Protesten gegen die Sparpolitik des Berliner Senats schreibt Fritz-Vannahme:

"Die Gesellschaft, bedrückt von den Sorgen ums Hier und Heute, verschwendet kaum einen Gedanken an die Zukunft, es sei denn es geht um die Renten. Und schließlich die Hochschulen selber, die alle Überlebensreflexe verloren haben und denen zur Revolte die Kraft, zur Reform das Rückgrat zu fehlen scheinen. Was sind schon 35000 Studenten und Professoren auf Berlins Straßen, gemessen am politisch geplanten Elend der dortigen Verhältnisse?« (Fritz-Vannahme, 1996, 1) Und weiter: «Wer als Student eine bessere Hochschule verlangt, muß sich Studiengebühren geradezu herbeiwünschen. Vernünftig und mit Maß eingefordert, nur zur Verbesserung der Hochschulen eingesetzt, gäben sie den Studenten ein Instrument der Mitsprache in die Hand.(..) Wer für seine Uni bezahlt, kann Qualität und Rechenschaft verlangen.« (ebd.)

Und wer nichts bezahlen kann, darf gar nichts verlangen und sollte den PragmatikerInnen bei ihrem Weg, der sie »mit Spaß durch die Institutionen« (Fritz-Vannahme 1995b, 47) führt, besser nicht im Wege stehen. Die Generation Student '95 soll das Unmögliche vollbringen: ihre KollegInnen rauswerfen, ohne ihnen wehzutun, Qualität von Forschung und Lehre verlangen, ohne nach ihrem Sinn zu fragen, und dabei Vernunft und Maß nicht aus den Augen verlieren.

\section{Links-Alternative und die gefährlich braven StudentInnen}

Wenn es richtig ist, daß die StudentInnen von heute mit Bündnis 90/DIE GRÜNEN die Partei ihres Vertrauens gefunden haben, dann ist die richtige Zeitung für beide die taz. Auch dort widmet man sich den Studierenden und ihren politischen Verpflichtungen seit einiger Zeit mit großer Aufmerksamkeit. Bereits 1994 forderte Michael Daxner, Präsident der Uni Oldenburg und seit Jahren rührig und rührend um die StudentInnen besorgt, man möge sie doch ernst nehmen, als politische PartnerInnen (Daxner 1994, 10). Die zukünftigen Auseinandersetzungsformen im Hochschulkonflikt umreißt Daxner wie folgt:

»Der Gesellschaftsvertrag zur Wissenschaft kann sich nur ais Generationenvertrag in der Bereitstellung der Mittel und als Studienvertrag in der Vermittlung darstellen. Die dafür notwendigen Mitbestimmungsformen lassen sich nicht durch eine Reparatur des Paritätenmodells herstellen, sondern nur aus einer Interessenallianz aller Hochschulangehörigen - Ernstnehmen, Vertrauen, aber auch Grenzen und Möglichkeiten der eigenen Kompetenz sind hier zu bestimmen. [...] Aus dem Konzept des Generationenvertrages ergibt sich, daß die Statusgruppenuniversität nicht mehr zeitgemäß sein kann. Im Hinblick auf die Organisation des Denkens und damit des Studiums könnte sich eine Verkehrsform des Konsenses einstellen.« (ebd.)

Dieses Bündnis für Kopfarbeit kommt hoffentlich nie zustande. Es scheiterte gewiß an einem Punkt, nämlich wenn es gilt, 50\% aller Stellen mit Frauen zu besetzen. Eher ziehen die Herren Akademiker - in dieser Frage vom Korpsstudenten bis zum marxistischen Professor Seite an Seite - in den bewaffneten Kampf, bevor der Männerdomäne Uni der Garaus gemacht werden kann. Wie schreibt Daxner: »Es gibt Studentinnen und Studenten « (ebd.). Kein Konsens drin. 
Gleichzeitig sollen die Studierenden der Öffentlichkeit gegenüber für die Uni und die Wissenschaft werben: Public relations in eigener Sache, oder doch nur für diejenigen, die der Institution ein Arbeitsleben lang verbunden sind - für die ProfessorInnen?

„Für die Öffnung der Hochschule zu dieser Öffentlichkeit, die für den Generationenvertrag einstehen soll, sind aber die Studierenden verantwortlich. Mit diesem Bewußtsein sollen sie zum Konsens über die Aufgaben und Funktionen der Wissenschaft in der Zukunft beitragen, besonders zu den Bedingungen, unter denen sie und die nächsten Generationen sich diese Wissenschaft aneignen werden.« (ebd.)

Ganz ähnlich wie Daxner, nur etwas dreister ging der ehemalige Berliner Wissenschaftssenator Ehrhardt zu Werke, der von 1991 - 1995 in der Hochschullandschaft des Landes plattmachte, was plattzumachen war. Kurz vor Dienstschluß, vor den Wahlen Ende 1995 ließ er dann eine Imagekampagne für die Hochschulen vom Stapel, »Bildung ist Zukunft Hochschulen bilden Berlin«. Angeblich sollte das »eine studentische Initative« sein. Eingefädelt hatte sie jedoch der Stifterverband für die deutsche Wissenschaft e.V. und »die Berliner Wirtschaft«. Ehrhardt, dem der Übergang vom Berliner Senat zu ebendiesem Stifterverband nahtlos gelang, war beleidigt und verwundert, daß mit Ausnahme des AStA der Hochschule der Künste keine Studierendenvertretung in Berlin Lust hatte, für den Chefabwickler die Werbefuzzis zu mimen. Auf einer Tagung der Deutschen Universitätszeitung (DUZ) im März 1996 in Bielefeld "Aus den Studien in die Medien - Studierende machen Hochschule öffenlich« machte Ehrhardt im Rahmen der Preisverleihung für studentische NachwuchsschreiberInnen nochmals deutlich, daß für ihn politisierte StudentInnen zuvörderst an der Marketingfront zu kämpfen hätten (Weise 1996, 12). Als ob er Daxner gelesen hätte. Dieser weiß natürlich, daß nicht jede StudentIn der Aufgabe gewachsen ist, die Universität zu repräsentieren, und legt als Zugangsvoraussetzungen fest:

»Auf dieser Grundlage kann allerdings erwartet werden, daß sich die Studierenden auch darüber klar werden, was sie von sich und der Hochschule zu fordern bereit und in der Lage sind: Präsenz, Verbindlichkeit in der Mitarbeit, regelmäßige Kritik der Veranstaltungen in deren Öffentlichkeit, Wahrnehmung von Beratungsangeboten, das sind die Aktionsfelder/-formen der Studierenden innerhalb der Hochschule und die Voraussetzungen, aus der abstrakten Forderungshaltung in eine 'Partnerschaft' mit der Institution zu gelangen.« (Daxner 1994, 10)

In einer Diskussion mit studentischen VertreterInnen von Freier Universität, Technischer Universität und Humboldt-Universität in Berlin im Juni 1996 (taz 25.6.1996), zeigt sich auch Peter Grottian, Professor am OttoSuhr-Institut, wenig zufrieden mit dem, was Studierende bisher im Rahmen ihrer Proteste für die Uni getan haben. »Die Studierenden müßten natürlich heftig Druck auf die Professoren ausüben, mit dieser Form der Umverteilung ihre eigenen Lehrbedingungen zu verbessern « (ebd.). »Diese Form der Umverteilung« meint ein von Grottian entwickeltes Modell, bei 
dem ProfessorInnen und der Mittelbau auf einen Teil ihrer Bezüge verzichten sollen. Mit diesem Geld sollen die existierenden Lücken gestopft werden und zusätzliche Mittel zur Ausstattung zur Verfügung stehen. Zu einem späteren Zeitpunkt sollen dann auch Studiengebühren von bis zu 250 Mark pro Jahr folgen (ebd.). Auf die Entgegnung des Studenten Jochen Geppert vom Kuratorium der FU, Profs tauchten ab, wenn sie politisch gefordert würden, meint Grottian: »Ihr habt sie nicht getreten. Ihr habt Diskussionen in den Seminaren geführt, aber die Frage, was das mit ihnen zu tun hat, ausgelassen« (ebd.). Grottian war es auch, der 1995 einen Aufruf zur Unruhe unter dem Titel $»$ Von einer Jugendrevolte... « verfaßte, der unter anderem in der Semesterbeilage der bereits erwähnten junge Welt veröffentlicht wurde. Grottian schreibt: »Der verdummende Unsinn, der die ideologische Grundlage hierfür [die jugendfeindliche Einsparpolitik] liefert, wird indes von den angeblich so kritischen jungen Leuten als marktwirtschaftliche Geschäftsgrundlage geschluckt und akzeptiert.« Trotzdem ist die Lage nicht hoffnungslos, denn:

»Glaubt man den seriösen sozialwissenschaftlichen Jugendstudien der letzten Jahre, dann haben die Jugendlichen ihr Gespür für gerechtere Macht- Arbeits- und Vermögensverhältnisse nicht verloren - aber die für Veränderungen nötige jugendliche Radikalität ist deutlich reduziert.«

\section{Der Weg zur Radikalität:}

»Wenn Jugendliche sehen, daß sich entlang ihrer Interessen wirklich etwas bewegt, werden sie das nicht nur am Fernseher verfolgen, sondern einer guten Tradition folgend, selbst in die Hand nehmen. Eine Jugendrevolte systematisch zu schüren ist Bürger- und Bürgerinnenpflicht« (Grottian 1995, 1). Kein Wunder, daß der grün-alternativen Sektion der HochschulmodernisiererInnen die Renitenz der Studis gegen die von ihnen erwartete Renitenz sauer aufstößt.

»Gefährlich brav? - Deutschlands StudentInnen haben keine Lust, die Welt zu verändern.« Unter dieser Überschrift stellt Martin Ebner in der taz im Juni 1996 eine Studie der Universität Konstanz vor (Ebner 1996, 11). WissenschaftlerInnen befragten im Auftrag des Bundesministeriums für Wissenschaft und Forschung im Wintersemester 1994/95 20.000 Studierende an 22 deutschen Hochschulen, »was sie von ihrem Studium, Gott und Europa halten« (ebd.). Einem der beiden Leiter der Studie, Tino Bargel, zufolge haben »die meisten Studierenden 'kein starkes allgemeines politisches Interesse und sind konventioneller und pragmatischer' als frühere Generationen« (ebd.). Die Mehrheit sei zwar noch immer rot-grün ausgerichtet, die Meinungsführerschaft hätten aber nicht mehr die SozialwissenschaftlerInnen, sondern die »meist brav und konservativ ausgerichteten Studierenden der Rechts- und Wirtschaftswissenschaften« übernommen (ebd.). Festzustellen seien geringeres Engangement und Distanzierung zu feministischen Forderungen, ebenso wie Mißtrauen gegenüber PolitikerInnen, Parteien und Parlamenten. Beruhigend stellt Ebner fest: »Direkt 
'rechts' sind die Studierenden aber auch nicht. Ausländerfeindliche Parolen und nationalistisches Gedankengut finden allenfalls bei Fachhochschülern in der Ex-DDR noch größeren Zuspruch.« Soso, also da und nur da kommen sie her. Immerhin $16 \%$ der StudentInnen seien bereit, sich »für mehr BAföG, eine Entrümpelung der Studiengänge, Verbesserungen der Lehre und mehr Hochschulpersonal in Aktionsgruppen zu engagieren." Das sind genau die, die mit den $84 \%$ aus Student '95 die Hundert voll machen. Im Grunde sind alle zu allem bereit. Trotzdem: Mitbestimmungsund Entscheidungsrechte in den Hochschulgremien kennt fast keiner, mit Ausnahme der Fachschaften. Der Beitrag schließt:

»Daß die Mehrheit der deutschen Studierenden sich nicht organisieren will und gar nicht daran denkt, 'das System zu sprengen', oder 'die Welt zu verbessern', dürften die meisten Politiker wohl beruhigend finden. Tino Bargel aber hält die 'Gleichgültigkeit' der Studentenschaft 'für gefährlich'. Der Hochschulforscher sieht hier 'Verantwortungslosigkeit, Egoismus und Mangel an Innovationsfreudigkeit'" (ebd.).

Eigenschaften, die im alternativen Lager dringend gebraucht werden, wie Verantwortung für die Umwelt, Gemeinsinn und der Mut, neue Wege in der Politik zu beschreiten, die Studis scheinen sie leider nicht zu besitzen.

\section{Konservative und die Suche nach den neuen Eliten}

»Steckt das Modell Deutschland in der Sackgasse? [...] Offenkundig ist, daß die Karre im Dreck steckt.« Wieder ist Die Zeit Ort der Besinnung, Constanze Stelzenmüller führt die Feder. Sie macht sich Gedanken über Roman Herzog und seine Ausführungen zum Thema Elite (Stelzenmüller 1996, 1). »Ohne leadership kann auch eine demokratische, pluralistische und offene Gesellschaft nicht existieren.Verständigen muß sie sich indes über die Kriterien der Auswahl.« Sehr vorsichtig und ausgewogen und unter dem Titel »Elite für alle« begibt sich die Autorin auf die Suche. Es fehlt nicht an Bedenken bezüglich der Verführbarkeit der Eliten in der Geschichte, ihrer tragenden Funktion für den Nationalsozialismus, ihrer Beteiligung an den Verbrechen. Und doch - die neue Elite soll ja nicht Verbrechen gegen die Menschheit planen und begehen, sondern nur die deutsche Karre aus dem Dreck ziehen. $»$ Wer kommt dann in Betracht? Die 'Leistungseliten', die Hochbegabten, wie es Roman Herzog fordert? Wahr ist so viel: Ohne die Fähigkeit, das Vorgegebene kritisch zu überprüfen und in neue Räume des Wissens vorzustoßen, kann keine Gesellschaft im globalen Wettbewerb überleben« (ebd.). Neue Eliten - eine Überlebensfrage?

Ende Juni 1996 hat Ulrich Greiner die Hamburger Uni besucht und dabei viele Stimmen und Meinungen eingefangen: von StudentInnen, vom Präsidenten Jürgen Lüthje, vom Wissenschaftssenator Leonhard Hajen und verschiedenen Professoren, unter anderem dem Anglisten Dietrich Schwanitz (Greiner 1996, 30). In seinem Uni-Krimi Campus, so Greiner, zeige 
Schwanitz »das Justemilieu aus linkem Besitzstandsdenken und Political Correctness, die Herrschaft aus Mittelmaß und Wahn« (ebd.). Etwas ändere sich, meint der Professor jetzt im Gespräch, er wisse nur nicht genau, was. Er sagt: »Vor Jahren noch mußte ein jeder, der nicht egalitär dachte und auf Leistung bestand, Gründe angeben und sich verteidigen. Heute ist es umgekehrt« (ebd.). Das verpönte Elite-Denken kehre zurück. Schwanitz spricht davon, daß der akademische Nachwuchs verramscht werde, es gebe zu wenig Druck auf alte Profs. Er hofft auf bessere Zeiten, wenn die Alt$68 \mathrm{er}$ endlich so alt sind, daß sie gehen müssen. Und der verramschte Nachwuchs? Sind die JungakademikerInnen für die großen Aufgaben bereit, die Stelzenmüller so vervollständigt:

»Aber im Deutschland von 1996 [...] bedarf es noch einer anderen Art von Elite: jener, für die Führung ohne Solidarität nicht denkbar ist; für die der Anspruch auf Rechte einhergeht mit dem Bewußtsein sozialer Verpflichtung; für die die Suche nach Spitzenleistungen das Streben nach Chancengleichheit einschließt; die das eigene Interesse im Licht des Gemeinwohl def1niert [...]. Man nennt das Verantwortungselite.« (Stelzenmüller 1996, 1)

Dieses Anforderungsprofil entspräche eigentlich dem Bild, wie es eingangs beschrieben Student '95 von der nachwachsenden Generation gezeichnet hat, aber leider scheint die Elite im Wartestand zurückzuscheuen: »Gefährlich brav? - Deutschlands Studenten haben keine Lust, die Welt zu verbessern." So steht's geschrieben im Allgemeinen Hochschulanzeiger der FAZ, Ausgabe Nr. 30 zum Sommersemester 1996. Der Autor heißt Martin Ebner und, wir ahnen es schon, er berichtet über eine Umfrage der Uni Kostanz, die wissen wollte, was Studis von Gott, ihrem Studium und Europa halten. Daß dieser Doppelabdruck ein Lapsus ist, der im Tagespressegeschäft schon mal passiert, damit aber die alte Phrase »von taz bis FAZ endlich einmal in quasi fleischlicher Gestalt Wirklichkeit werden läßt, ist eine amüsante Nebensache, außer vielleicht für die Leute vom Marketing, die die 5000 neuen Abos für die taz mit deren absoluter Einmaligkeit begründen. Entscheidend ist hier, daß die Enttäuschung über die unpolitischen StudentInnen sich jetzt ganz anders decodieren läßt. Ein nicht ganz unwesentlicher Passus fehlt neben anderen Details in der taz-Version:

»Satte 59 Prozent der Wessis - vier Prozent mehr als 1992! - erklären explizit, keine Kontakte mit Kommilitonen aus 'Neufünfland' zu wünschen. Nur rund ein Prozent denkt an einen Hochschulwechsel in den Osten - sehr viel geringer ist der Austausch mit Afrika auch nicht. Die Mehrheit der ostdeutschen Studenten wiederum hat wenig Lust, sich am Bau des europäischen Hauses zu beteiligen, viele befürchten den 'Verlust nationaler Identität' und 'kulturelle Überfremdung'. Auch im Westen stößt die Binnenmarktpropaganda vom großen Wohlstandsgewinn auf Skepsis. Nur die Juristen und Wirtschaftswissenschaftler«, die neuerdings den Ton angeben, »sehen dem europäischen Arbeitsmarkt hoffnungsfroh entgegen. Viele Studierende haben Angst vor wirtschaftlicher Instabilität und einer zentralistischen Eurokratie in Brüssel.« (Ebner 1996, 29)

Auch bei der FAZ schließt der Beitrag mit Bargels Hinweis auf die »gefährliche Gleichgültigkeit« der StudentInnen, ihre »Verantwortungslosigkeit, Egoismus und Mangel an Innovationsfreudigkeit«. Jetzt liest sich 
das allerdings als die Forderung an die StudentInnen, ihre Rolle als Verantwortungselite der europäischen Hegemonialmacht nicht zu verweigern, sich mehr für die Einheit zu interessieren und dafür Opfer zu bringen, und bei Investitionen und beim Abbau sozialer Besitzstände Risikofreudigkeit zu zeigen. StudentInnen '96 - nie sollst Du sie befragen.

\section{Studentische Politik}

Soweit mit Aufmerksamkeiten überhäuft, agieren die StudentInnen im öffentlichen Raum. Wie reagieren sie auf all die Erwartungen, die man in sie steckt? Vor allem versucht ihr aktiver Teil in den letzten Jahren verzweifelt eines: studentische Politik zu machen. Dadurch wurden sie erst recht unpolitisch, denn "studentische Politik« gibt es nicht. Es ist der Versuch, völlig disparate Interessen unter einem ständisch geprägten Begriff zusammenzufassen, gepaart mit der tiefen Sehnsucht, politikfähig zu sein. Die AktivistInnen wollen teilhaben dürfen am Spiel der Kräfte, in Verhandlungsrunden mit den üblen PolitikerInnen und der Hochschuladministration. Die politische Stärke dafür, so war es bisher gedacht, sollte durch eine Massenbewegung hergestellt werden. Im Sommer 1994 beispielsweise mobiliserte der $f z s$, der freiwillige zusammenschluß von studentInnenschaften, für eine bundesweite Großkundgebung »Gegen Sozialabbau und Bildungsklau « in Bonn und rechnete mit 10.000 TeilnehmerInnen. Als etwa 1000 kamen, waren die StudentInnenfunktionäre arg ernüchtert. Als im August 1995 die BAföG-Pläne von Zukunftsminister Rüttgers bekannt wurden, drohte der $f z s$ in DGB-Diktion mit einem »heißen Herbst«, der mangels Masse dann eher lau ausfiel. Seit dem Beginn des Sommersemsters versucht eine relativ kleine Gruppe von AktivistInnen an den Berliner Hochschulen ihre KommilitonInnen zum Mitmachen bei ihren Protestaktionen zu bewegen. Ein Streik war bisher an keiner Uni mehrheitsfähig, auf die wöchentlich stattfindenden »Montagsdemos«, der Name sollte offenbar für durchschlagenden Erfolg garantieren, verloren sich nur ausnahmsweise mehr als ein paar hundert StudentInnen.

In einer Broschüre, die Anfang Januar von »Menschen, die sich um Befreiung bemühen« am »befreiten OSI«, dem Otto-Suhr-Institut für Politikwissenschaft an der FU, veröffentlicht wurde, schreibt ein/e Unbekannte/r repräsentativ für viele andere Äußerungen:

>Um [Druck von außerhalb der Strukturen] erfolgversprechend ausüben zu können, bedarf es einer überzeugend großen Gruppe Aktiver. Die machtvolle und geschlossen agierende Gruppe »die Studierenden« gibt es nicht. Abgesehen von ihrer Medienwirksamkeit, sind Aktionen, wie die Besetzung der Rostlaube, nicht zuletzt auch der Versuch, vor sich hin dümpelnde Studis aufzurütteln und denen, die zwar unzufrieden, aber ohne Perspektiven sind, Wege und Aktivitäten aufzuzeigen. Die Hoffnung der 'Radikalen' ist dabei, daß wir uns zusammenschließen und endlich gemeinsam handeln.Hätten wir wirksame legale Einflußmöglichkeiten, wäre diese 'Radikalität' überflüssig. « Weiter heißt es: »Die einheitliche Gruppe aller Studie- 
renden ist natürlich utopisch und auch nicht wünschenswert. Daß wir mehrheitlich geschlossen handeln, ist aber Grundvoraussetzung dafür, daß sich irgendwas in unserem Sinne bewegt.« Die AutorIn fordert: »Wir müssen zu den Akteurinnen und Akteuren werden! Das müßte vor allem Politik-Studierenden, die schließlich jeden Tag auf der Suche nach der herrschaftsfreien Gesellschaft sind, einleuchten...« (Nutzlos und gemein 1996, 18)

Sind die StudentInnen am OSI jeden Tag auf der Suche nach der herrschaftsfreien Gesellschaft? Das OSI, Symbol für die »linke Uni«, war in den letzten Jahren verschiedentlich Gegenstand der Hochschulberichterstattung der Süddeutsche Zeitung. 1994 kommen unter der Überschrift. $»$ Am Berliner Otto-Suhr-Institut - einst Hochburg der Studentenbewegung - will man von den Ideen der 68er nichts mehr wissen« verschiedene ProfessorInnen zu Wort:

»Weit entfernt von [den Niederungen der Tagespolitik] sind auch die gern als angepaßt und stromlinienförmig charakterisierten Studentinnen und Studenten der neunziger Jahre. 'Ja, braver und höflicher' seien sie geworden, meint die Dekanin [damals Gesine Schwan, R.O.], 'und die Tendenz zum Einzelkämpfertum ist eindeutig da.' Von Kollektivbewußtsein sei nicht mehr viel übrig. 'Entpolitisiert' worden seien die Studenten am Institut, weil sie 'jahrelang irregeleitet waren und ihnen politisch utopische Forderungen vermittelt wurden', merkt Fritz Vilmar an, seit über 25 Jahren am Institut.[...] Das Hohe Lied der 68er auf 'Respektlosigkeit, Liberalität und Freiheit' ist verstummt. « Eine ältere Studentin meint über die neuen Jahrgänge: "'Die wissen knallhart was sie wollen: in den internationalen Dienst oder zu den Medien.« (Mahnkopf, SZ 1994)

Im Mai 1995 wird an gleicher Stelle berichtet, daß am OSI wieder feierliche Abschlußveranstaltungen und Diplomverleihungen stattfinden würden - als erster Fachbereich der FU und auf audrücklichen Wunsch der StudentInnen. »Fast zwei Drittel aller geladenen Studenten waren erschienen« (von der Osten 1995). Kurz gesagt, »die anderen StudentInnen«, die zusammen mit den AktivistInnen die herrschaftsfreie Gesellschaft bauen sollen, sie kommen nicht mehr. Sie müssen auch nicht mehr mobilisiert werden. Es hat praktisch keine studentische Publikation und Position in den letzten zwei Jahren gegeben, die sich nicht auf die Suche nach »studentischer Politik « begeben hätte ${ }^{2}$. Dieser Begriff suggeriert eine Einheitlichkeit studentischer Anliegen, die so nie existiert hat, und lockt mit Politik in der ersten Person. Wir, die Studis. Gleichzeitig ist er die Einladung an die Gegenseite, konservative Profs und technokratische PlanerInnen, brenzlige Situationen an der Uni einfach auszusitzen. Auch renitente Studis sind irgendwann keine Studis mehr. Studentische Politik ist wie Olympiade: alle vier Jahre, und hinterher war's toll, wenn man dabei war.

2 Das bereits erwähnte unikonkret nennt als MitherausgeberInnen 19 linke Asten und studentische Gruppen, darunter das Kollektiv der Liste LINKS an der Uni Hamburg. Es schreibt: »Vollständige Demokratisierung über den sozialen Prozeß steht auf der historischen Tagesordnung. Studentische Politik als Teil gesellschaftlicher Opposition hat hieran mitzuwirken (Liste LINKS 1996, 21). Die Liste LINKS hat auch »Thesen für eine fortschrittliche studentische Wissenschafts- und Gesellschaftspolitik« verfaßt. Das ist die einzige Position von StudentInnen zu dieser Frage in unikonkret. 
Nicht nur, weil im Juni '96 in Berlin Aktionsformen wie ein 24-StundenRennen am Ernst-Reuter-Platz und ein Eierlaufen zum Sitz des Wissenschaftssenators (»StudentInnen geben den Löffel ab《) zu bewundern waren. Um dauerhaft die Voraussetzungen für gesellschaftliche Veränderungen und so für eine andere Uni zu schaffen, sollte endlich die Behauptung einer genuin studentischen Politik ersetzt werden durch den Blick auf die Hochschulen in ihrer Funktion im ökonomischen und ideologischen Gefüge der BRD als Ganzes. Nicht der Grad der eigenen Betroffenheit ist maßgebend, sondern die Wichtigkeit der Hochschulen im System. Was immer an den Hochschulen im emanzipativen Sinne verändert werden soll: Es wird nur von einer Minderheit in Uni und Gesellschaft getragen.

»Mit ihrem 'Leistung lohnt sich wieder'-Gelaber in den Achtzigern und der angestachelten 'Standort Deutschland' Hysterie der Neunziger, verbunden mit dem massiven Sozialabbau, ist es konservativen Politikern und Wirtschafts-Funktionären gelungen, die Gesellschaft und den Konkurrenzkampf unter einander voranzutreiben« (Nutzlos und gemein 1996, 19).

Auch wenn es verführerisch ist, das Böse in der Welt den PolitikerInnen in die Schuhe zu schieben: Sie wurden unter anderem von StudentInnen gewählt, um deren konservative Vorstellungen umzusetzen. Die jetzt aktiven StudentInnen kommen nicht daran vorbei, ihre Vorstellungen von einer besseren Uni gegen die Mehrheit ihrer KommilitonInnen zu entwickeln, wenn sie Erfolg haben wollen. Denn die meisten sind mit dem zufrieden, was der Staat noch bereit ist, in Zukunft zu bieten: eine gute berufliche Qualifikation, wenn es sein muß, auch privat finanziert. Keine Gesellschaftskritik, keine Bildung als Grundrecht für alle, keine Erprobung anderer wissenschaftlicher Ansätze. Wenn in Zukunft weniger studieren, bedeutet das nur verbesserte Chancen für die, die weiterhin an der Uni bleiben können. Wenn Firlefanz wie feministische Wissenschaft, Marxismus und Ideologiekritik verschwinden, dann bleibt mehr Geld für die wichtigen Dinge: Germanistik? Ich lese nur zur Entspannung! Kapital-Kurs? Geh doch nach drüben! Rassismusforschung? Mein Freund ist Ausländer!

Ebenfalls illusorisch ist es zu glauben, man hätte »radikale« Aktionen wie die kurzzeitige Besetzung des OSI nicht nötig, wenn man »wirksame legale Einflußmöglichkeiten« hätte. Es ist zwar richtig, daß die StudentInnen im Modell der Gruppenuniversität weniger zu sagen haben als die ProfessorInnen, aber selbst die Spielräume, die sie haben, nützen sie längst nicht aus. Wieviel Mühe ist es immer, all die Gremienplätze, die für StudentInnen vorgesehen sind, mit kompetenten Leuten zu besetzen? Wie sehr hängt die politische inhaltliche Arbeit an einigen wenigen, weil sich fast niemand für Hochschulpolitik interessiert und weil keine StudentInnengeneration in der Lage ist, ihr politisches Wissen weiterzugeben? Ihr Recht, ihre politische Vertretung, den AStA, selbst zu bestimmen, nehmen seit Jahren nur etwa 10 Prozent der Studierenden wahr. Zu einer Diskussion mit der Berli- 
ner Finanzsenatorin Fugmann-Heesing Anfang Juli im Audimax der FU kamen 13 Studierende (dreizehn!) (taz, 5.7.1996).

Mittlerweile haben sich erste Zweifel am Konzept einer Massenbewegung breitgemacht. Nicht nur wegen des bevorstehenden Semesterendes hat die Beteiligung an den Aktionen in Berlin Ende Juni/Anfang Juli stark nachgelassen. Wesentlich effektiver scheint es jetzt, den Boykott der "Verwaltungsgebühr« in Höhe von hundert Mark zu organisieren, die ab dem Wintersemester 1996/97 erhoben werden soll (»Ohne Hunni in die Uni«). Daß diese Maßnahme tatsächlich die Einführung von Studiengebühren bedeutet, wissen alle. Und über ein Drittel derjenigen, die bisher ihre Rückmeldungsgebühr bezahlt haben, haben die hundert Mark nicht bezahlt. Die OrganisatorInnen hoffen, diesen Beteiligungsgrad halten zu können. Außerdem rechnet sie mit einer Klagewelle, da nach einem Gutachten der GEW die Erhebung der Gebühr rechtswidrig sein soll (taz, 28.6.96). Mehr ist zur Zeit nicht drin, scheint es. Im Wintersemester soll es dann weitergehen. Wie, weiß noch keiner so richtig. In den letzten Wochen haben sich zahlreiche studentische Gruppen gebildet, die inhaltlich arbeiten wollen. Viele haben gemerkt, daß die Aktionen zwar phantasievoll sind und viel Aufmerksamkeit und Wohlwollen genießen, doch die politischen Details kennen die wenigsten. Wie funktioniert ein Uni-Etat? Was kostet ein Studienplatz und wie werden die Kosten berechnet? Was ist der Wissenschaftsrat und wieso hat er soviel Einfluß? Sie wollen es wissen. Nicht mehr, um ihre Mitstudierenden aufzuklären, sondern für sich selbst. Und dann? Natürlich ist die Verlockung groß, nach der Sommerpause mit eigenen Reformkonzepten aufzuwarten. Aber läuft man damit nicht genau in die realpolitische Falle, in der die Oppositionsparteien oder Verbände auch landen, wenn sie sich an angeblichen Parametern für Machbarkeit orientieren, wie der DGB mit seinem arbeitnehmerInnenfreundlichen Kurs? Im Bezug auf die Uni ist das Beispiel der BAföG-Diskussion aussagekräftig: Am 28. Juni hat der Bundestag zwar das Vollzins-Modell von Rüttgers vorerst begraben (taz 29/30.6.1996). Der Ersatz, Verzinsung des Darlehensanteils nach Überschreitung der Regelstudienzeit, ist aber zum Studieren zu wenig, zum Sterben zu viel. Im letzten dreiviertel Jahr haben alle Parteien, dazu die $G E W$, das Studentenwerk und der $f z s$ Gegenvorschläge gemacht. Und alle haben sich an der Vorgabe Kostenneutralität orientiert. Bei Bündnis 90/DIE GRÜNEN setzte sich intern das BAFF-Modell einer Ausbildungskasse von Mathias Berninger gegen das Konzept von Elisabeth Altmann durch. Letzteres war das einzige von acht BAföG-Modellen, bei dem offen mehr Geld gefordert wurde ${ }^{3}$. Es verschwand in der Schublade.

3 Zum BAFF vgl. Oberndörfer $(1996,56)$. Einen guten Überblick über alle BAföG-Modelle findet sich bei Weitkamp $(1996,52)$. Weitkamp ist Mitarbeiter im Büro von Elisabeth Altmann. 


\section{Andere Ansätze}

Neben den hier skizzierten Unerfreulichkeiten gibt es wenigstens zwei Ansätze, die vielleicht mittelfristig Perspektiven beinhalten können, die über das reine »Politik machen « hinausgehen. Zum einen den bereits erwähnten freien zusammenschluß von studentInnenschaften $(f z s)$, zum anderen das Bündnis gegen Sozialabbau in Berlin. Der $f z$ ist eigentlich ein Paradox. Er ist ein Zusammenschluß von etwa 70 Asten, StuRäten und anderen StudentInnenvertretungen, von denen es etwa 300 in der BRD gibt. Nach dem Ende der vds (vereinigten deutschen studentInnenschaften) 1990 (Korb $1990,1)$, ist er die erste studentische Organisation, die parteiunabhängig auf Bundesebene arbeitet. Der $f z s$ ist hervorgegangen aus dem studentischen Bildungsgipfel 1993 in Bonn (Oberndörfer 1993, 2), und hatte einen Vorläufer in dem Zusamenschluß studentischer Vertretungen an Fachhochschulen (FKS). Der $f z s$ gibt etwa vierteljährlich einen Rundbrief heraus, den nicht nur Mitglieder beziehen können. Er war Mitorganisator des studentischen Festivals Aufbruch 95 in München (Berth 1995, 4). Zum einen betreibt der $f z s$ klassische Lobbyarbeit. In der BAföG-Diskussion war er sehr aktiv, veröffentlichte zahlreiche Presseerklärungen und organisierte Veranstaltungen, auf denen sich ExpertInnen in seinem Sinne äußerten. All das läuft, wie gehabt, unter der Überschrift studentische Politik. Daß der $f z s$ als Organisation von FuntionärInnen bei den bekannten Wahlbeteiligungen fast keine Basisanbindung hat, daß bei siebzig verschiedenen Asten, die jedes Jahr neu gewählt werden, eine einheitliche Strategie über minimale Forderungen im sozialen/technischen Bereich hinaus fast nicht möglich ist, daß der $f z s$ nur eine Minderheit der Studierenden repräsentiert, weil viele große Asten keine Lust haben, sich von kleinen Asten mitregieren zu lassen und dafür auch noch teure Mitgliedsbeiträge zu zahlen - all das störte die MacherInnen bisher wenig. In einem haben sie recht: Gegenforderungen gehören auf die Bundesebene. Regionale Streitereien, im Bundesland, oder womöglich noch in der Uni oder im Fachbereich, bringen bei grundsätzlichen Fragen, insbesondere Kürzungen, fast nichts. Bonn ist die richtige Adresse, dort hat der $f z s$ seinen Sitz.

Gleichzeitig zu seiner Lobbyarbeit hat der $f z s$ ernsthaft vor, fundamentale Systemkritik zu üben und über die Tagespolitik hinauszugehen. Der Rundbrief Nr. 20 vom Mai 1996 hat als inhaltlichen Schwerpunkt zum Beispiel »Rassistischer Sozialstaat«, erarbeitet vom $f z s$-eigenen Arbeitskreis zu diesem Thema. Darin finden sich Beiträge zur sozialpolitischen Ausgrenzung von AusländerInnen, ein Text von Oskar Negt zu »Restriktionen des Asylrechts und soziale Krise«, ein Überblick über Arbeits- und Ausbildungsverbote von AusländerInnen und vieles mehr, was die Mehrheit der StudentInnen sicher keinen Deut interessiert. Auf dem Kongreß in Bonn 1993 
hieß es in einem vorbereitenden Papier zur Uni-Reform: »Die Inhalte von Forschung und Lehre müssen sich an antirassistischen, antisexistischen, ökologischen und sozialen Grundsätzen orientieren« (Bildungsgipfel 1993, 23) Platt und ungeschlacht wie ein autonomes Flugblatt tönt das, aber gleichzeitg angenehm utopisch mitten in so viel Realpolitik. Wie der $f z s$ es schaffen will, »studentische Interessen « zu vertreten und gleichzeitig marginale Positionen wie feministische Wissenschaft und Antirassismus zu stärken, wissen wahrscheinlich nicht mal seine VertreterInnen.

Seit Dezember 1995 hatte man in Bonn eine Demo gegen Sozialabbau vorbereitet und dazu das »Bündnis Sternmarsch gegen Sozialabbau《 mitgegründet. »Wir haben den Sternmarsch nie als spezifisch studentisches Projekt begriffen, sondern von Beginn an versucht, sämtliche Gruppen einzubeziehen.« So zitiert die taz Jörg Prante vom Asta der Uni Bonn (taz 15/16.6. 1996). »Sämtliche Gruppen« hieß unter anderem auch kritische GewerkschafterInnen, die spätestens seit der 1. Mai-Demo in Berlin die Unser-Standort-soll-schöner-werden-Politik von DGB-Chef Schulte und anderen FunktionärInnen nicht mehr mitmachen wollen. Der DGB, dem die Idee zu seiner Großkundgebung am gleichen Tag erst viel später einfiel, deckelte kraft Masse die widerborstige Sternmarsch-Gruppe einfach zu. Die OrganisatorInnen durften die ihnen zugesagte Infrastruktur, u.a. eine Bühne nicht mehr benutzen. Weil auf der Demo plötzlich DGB draufstand, glaubt Prante, seien die StudentInnen nicht mehr so ansprechbar gewesen wie erhofft. Trotzdem, studentische Politik ist das nicht mehr. Was es sein könnte, hängt nicht zuletzt von den kritischen GewerkschafterInnen $\mathrm{ab}$ und davon, wie erfolgreich der $f z s$ bei seinem Balanceakt ist.

Die Idee von einem gemeinsamen Bündnis gegen Sozialabbau, an dem auch StudentInnen sich beteiligen, kam etwas früher bereits in Berlin auf. Dort konnte eine gleichnamige Initiative im Dezember 1995 über 10000 , im Frühjahr 1996 mehr als 35000 Leute mobilisieren. Es war dies eine der größten Demos der letzten Jahre in Berlin. Auf den ersten Blick scheint es auch überzeugend, wenn StudentInnen zusammen mit den anderen, die durch die Umstrukturierung des Sozialstaats besonders bedroht sind, mit Arbeitslosen, SozialhilfeempfängerInnen und Flüchtlingen, gemeinsam demonstrieren wollen. Alle, die aus unterschiedlichen Gründen in einem Abhängigkeitsverhältnis zum Staat stehen, sind irgendwie betroffen. Also ist es nur konsequent, wenn alle zu einer Demonstration aufrufen: Gemeinsam sind wir stark, unsere Anliegen sind ähnlich strukturiert. Wenn Flüchtlinge und StudentInnen sich gemeinsam gegen staatliche Maßnahmen zur Wehr setzen schafft das Solidarität. Wenn aber, wie in dem Aufruf zur Demonstration am 14. Dezember $1995 »$ Polizeiterror« gegen AusländerInnen mit der Einführung von leistungsorientierten Studienstrukturen in einem Atemzug genannt wird, werden fundamentale Unterschiede ver- 
wischt. Eine mißglückte Klausur oder eine Zwangsberatung kann einem den Tag verderben, eine Abschiebung kann das Leben kosten.

So, wie der Demoaufruf vom Dezember abgefaßt war, ging auch verloren, daß Studierende in realer ökonomischer Konkurrenz zu den anderen bedrohten Gruppen stehen, und dabei bessere Karten haben als eine Langzeitarbeitslose oder ein Flüchtling. Die meisten StudentInnen jobben, weil sie müssen, weil sie der Zwang der Reproduktion dazu nötigt, nicht wie Fritz-Vannahme unterstellt, um ihre Lebensqualität zu steigem (FritzVannahme 1995b, 48). Gerade aber, weil objektive Umstände sie dazu nötigen, sieht es ein bißchen hilflos aus, wenn die StudentInnen nicht aufhören zu beteuern, sie wollten sich nicht gegen die Marginalisierten ausspielen lassen. Wer seine Nischenexistenz als JobberIn mit Immatrikulationsbescheinigung gefunden hat, und in ungesicherten Arbeitsverhältnissen tätig ist, wer sich sein Studium selbst finanziert, trägt zur Erodierung von Stammbelegschaften, Lohndrückerei und Flexibilisierung bei. Der gute Wille spielt dabei keine Rolle. Und jedeR, wirklich jedeR, hat die Gewißheit, später »etwas anderes « machen zu können, nicht als Putze, als Bierfahrer, auf dem Bau oder in der Kneipe. Es soll nicht bestritten werden, daß das Leben als (arbeitslose) AkademikerIn schwierig ist, für andere ist es ungleich schwieriger.

Bevor sich die StudentInnen mit den Marginalisierten verbünden, müßten sie herausarbeiten, was sie von diesen trennt. Sie sind auf dem Weg zu einer überdurchschnittlichen Qualifikation, die ihnen hilft, irgendwo ein einigermaßen lukratives und interessantes Auskommen zu finden. AkademikerInnen wird es als allerletzten schlecht gehen. Der Standort Deutschland braucht sie. Flüchtlinge und SozialhilfeempfängerInnen braucht niemand. Sie sind nach kapitalistischen Maßstäben überflüssig, und überflüssig zu sein, ist tendenziell lebensgefährlich.

$\mathrm{Zu}$ einer Kritik der eigenen Interessenpolitik im Rahmen der Diskussion über Sozialleistungen gehört auch eine Kritik des Widerparts, des Staates. Im Kampf um den Erhalt seiner sozialen Aufgaben geht es fast immer nur um den Staat als Geber. Das Soziale wird eingefordert, mit Bezug auf das Grundgesetz oder aus humanitären Gründen, gelegentlich verbunden mit der Drohung oder Hoffnung, mit der sozialen Frage werde auch bald wieder die sozialistische Frage auf der politischen Agenda stehen. Von wem man fordert, und was die Erfüllung solcher Forderungen bedeutet, bleibt meist unhinterfragt. Es ist der gute, der gütige, der gewährende Vater Staat. Niemand, von dem man sich unbedingt etwas schenken lassen möchte, wenn man das System umkrempeln will. Es fehlt den StudentInnen hier an einer Kritik der staatlichen Sozialpolitik (Fraser/Gordon 1993, 322). Wer Leistungen vom Staat erhält, verbündet sich mit ihm. Und der Staat gibt nichts umsonst. Die finanzielle Unterstützung ist gekoppelt an die Verfüg- 
barkeit der Unterstützten. Für Arbeitslose bedeutet das einen immer massiver werdenden Arbeitszwang, für StudentInnen die Pflicht, durch immer absurdere Leistungsnachweise die Berechtigung zum Geldempfang nachzuweisen. Das BAföG trug zwar wesentlich zur Öffnung der Hochschulen in den Siebzigern bei. Es sorgte aber auch dafür, daß viele der für den Staat bedrohlich scheinenden StudentInnen von ihm versorgt und dadurch mittelbar kontrolliert wurden.

Je nach Kontext kann aus einer fortschrittlichen Forderung plötzlich eine reaktionäre werden, zum Beispiel die, das BAföG als Zuschuß auszuzahlen. Im Rahmen der BAföG-Diskussion bedeutet die Forderung: Bildung für alle, und das kostenlos. Im Kontext der Sozialstaatsdiskussion wird daraus plötzlich die Forderung, als StudentIn vor allen anderen bedient zu werden und möglichst ungestört - ohne Reproduktionszwang - seine faire Aufstiegschance zu bekommen, Abhängigkeit inbegriffen. Das Bündnis gegen Sozialabbau ist der interessanteste Ansatz von allen, aber bisher tun sich die verschiedenen Gruppen noch schwer, eine gut fundierte Plattform zu bauen. Studentische Politik jedenfalls hat keine Perspektive.

\section{Was fehlt, ist Wissenschaftskritik}

Was die StudentInnen erstaunlicherweise bisher überhaupt nicht interessiert, ist die Kritik ihres eigentlichen Metiers, der Wissenschaft. Das, was immer Bewegung in die Hochschulen brachte, der Versuch, die Frage: Wozu und zu welchem Ende studiere ich? neu zu beantworten, sie spielt im Augenblick keine Rolle. Sie enthält im Grunde alle anderen Fragen: Was ist der herrschende Wissenschaftsbegriff und wieso ist er so geworden? Was für ein Interesse hat der Staat an meiner wissenschaftlichen Ausbildung? Wer darf lehren? Wer darf lernen? Wer darf forschen? Was wird finanziert? Die letzte Gelegenheit, sich mit diesen zentralen Fragen auseinanderzusetzen, haben die StudentInnen im Westen verpaßt, als sie die Abwicklung im Osten als regionales Problem glaubten, ignorieren zu können. Niemand hörte auf die wenigen kritischen Stimmen, die den Kahlschlag der Ostwissenschaften als Probe aufs Exempel für die Westunis deuteten. Heute versucht der Staat auch im Westen neu zu definieren, was taugliche Wissenschaft ist und was nicht. Diesmal macht er es nicht mit Kriterien, die dem ideologischen Gegensatz zwischen Ost und West entspringen, sondern ganz objektiv und unideologisch, mit Hilfe des Geldes und der Frage nach Nützlichkeit.

Heute geht es (auch) den Westunis an den Kragen, aber obwohl mittlerweile StudentInnen in Ost- und Westberlin stärker zusammenarbeiten als noch vor drei oder vier Jahren, findet eine Kritik der Uni als Institution des Staates und damit des Staates selbst kaum statt. Die oben zitierte Frage 
nach dem Sinn und Zweck eines Studiums heißt die Frage nach den Interessen zu stellen, denen das Studium unterworfen ist. Die verschiedenen gesellschaftlichen Interessen an einer bestimmten Sorte von StudentInnen wurden im ersten Teil des Beitrags dargestellt. Es ist seltsam, wie gerne StudentInnen über studentische Politik reden und wie ungern jedeR für sich seine/ihre Interessen bestimmen will, wie sehr sie sich von allen Seiten zurechtdefinieren lassen. Wenn die aktiven StudentInnen anfingen, ihre Interessen zu benennen, kämen sie nicht daran vorbei, Staat und Uni einer radikalen Kritik zu unterziehen. Sie würden feststellen, daß sie Interessen haben, die nicht die der ProfessorInnen, nicht die der Hochschule, nicht die der Mehrheitsgesellschaft, nicht die ihrer MitstudentInnen sind. Es wäre das Ende von studentischer Politik und der erste Schritt hin zu einer politisierten Studierendenschaft.

\section{Literatur}

Anonym: B-Freite Uni - Alles, was wir tun ist nutzlos... aber unheimlich gemein, ca. Januar 1996, 20 Seiten, ohne Paginierung (zitiert: Nutzlos und gemein).

Berth, Felix (1995): Nachfrage nach Studiengängen, die "sich lohnen«, in: taz, 17/18.8.1995, S. 4.

Bojadzijev, Manuela (1994): Wie rechts sind die Studis?, in: FAUST 94/2, S. 3-4.

Daxner, Manfred (1994): Vereinbaren, nicht nomieren!, in: taz 4.5.1994, S. 10.

Ebner, Martin (1996): Gefährlich brav?, in: taz, 24.Juni 1996, S. 11, sowie in: Allgemeiner Hochschulanzeiger der FAZ, Nr. 30, SoSe 1996, S. 29.

Fraser, Nancy; Gordon, Linda (1993): Dekodierung von »Abhängigkeit«, in: Kritische Justiz 1993, S. $306-323$.

Fritz-Vannahme, Joachim (1995a): Die optimistische Generation, in: Die Zeit Nr. 43/95, 20.10.1995, S. 41-43.

- (1995b): Mit Spaß durch die Institutionen, in: Die Zeit Nr.44/95, 27.10.1995, S. 47-49.

- (1996): Humboldts Erbe wird verspielt, in: Die Zeit Nr.21/96, 17.5.1996, S. 1.

Greiner, Ulrich (1996): Es geht voran. Es geht, in: Die Zeit Nr. 27/96, 28.6.1996, S. 30.

Gremliza, Hermann L: Vergeßt 68!, in: unikonkret Sommersemster 1996, S. 1-2.

Grottian, Peter (1995): Von einer Jugendrevolte..., in: junge Welt Universität, Beilage am 18.10.1995, S. 1.

Grunenberg, Nina (1995): Und der Zukunft zugewandt, in: Die Zeit Nr.43/95, 20.10.1995, S.1.

Kohr, Knud (1996): Genauso dumm wie ich, in: unikonkret Sommersemster 1996, S. 2.

Korb, Stephan (1990): Das lange Sterben der vds, in: FAUST Nr. 5/90, S. 1.

Liste LINKS (1996): Grüne Wege, in: unikonkret Sommersemester 1996, S. 21.

Mahimann, Matthias (1994): Öffnung und Restauration. Zur sozialen Herkunft der Studierenden, in: Forum Recht, 1994, S. 56-57.

Mahnkopf, Cordula (1994): Die normative Kraft des Faktischen, in: Süddeutsche Zeitung 26./27.11. 1994.

Materialien zum Bildungsgipfel 1993 (1993), (zitiert: Bildungsgipfel).

Oberndörfer, Ralf (1993): Das war der Gipfel! in: FAUST Nr. 3/93, S. 2.

- (1996): Studium als Privatvergnügen, in: Fortum Recht 2/96, S. 56.

Otte, Carsten (1996): Studentischer Schreibzwang, in: unikonkret Sommersemester 1996, S. 23.

Ripplinger, Stefan (1996): Drei Wörter, in: unikonkret Sommersemester 1996, S. 5.

Stelzenmüller, Constanze (1996): Elite für alle, in: Die Zeit Nr.24/96, 7.6.1996, S. 1.

Student '95, Umfrage von Infratest Burke Berlin (1995a und 1995b), in: Die Zeit Nr. 43/95, 20.10.1995, S. 41-43 und Die Zeit 44/95, 27.10.1995, S. 47-49. 
von der Osten, Ernestine (1995): Händedruck am Hinterausgang, in: Süddeutsche Zeitung, 15.5.1995.

Walther, Christian (1996): Protest und Perspektiven, in: FU-Nachrichten 5/96, 15.Mai 1996.

Weise, Det (1996): Sehn wir uns nicht in dieser Welt, in: FAUST 96/1, S. 12.

Weitkamp, Rolf (1996): Kostenneutralität als Konsens, in: Forum Wissenschaft 2/96, S. 52.

Zimmer, Dieter E. (1996): Wenn das Humboldt wüßte..., in: Die Zeit Nr. 20/96, 10.5.1996, S. 35.

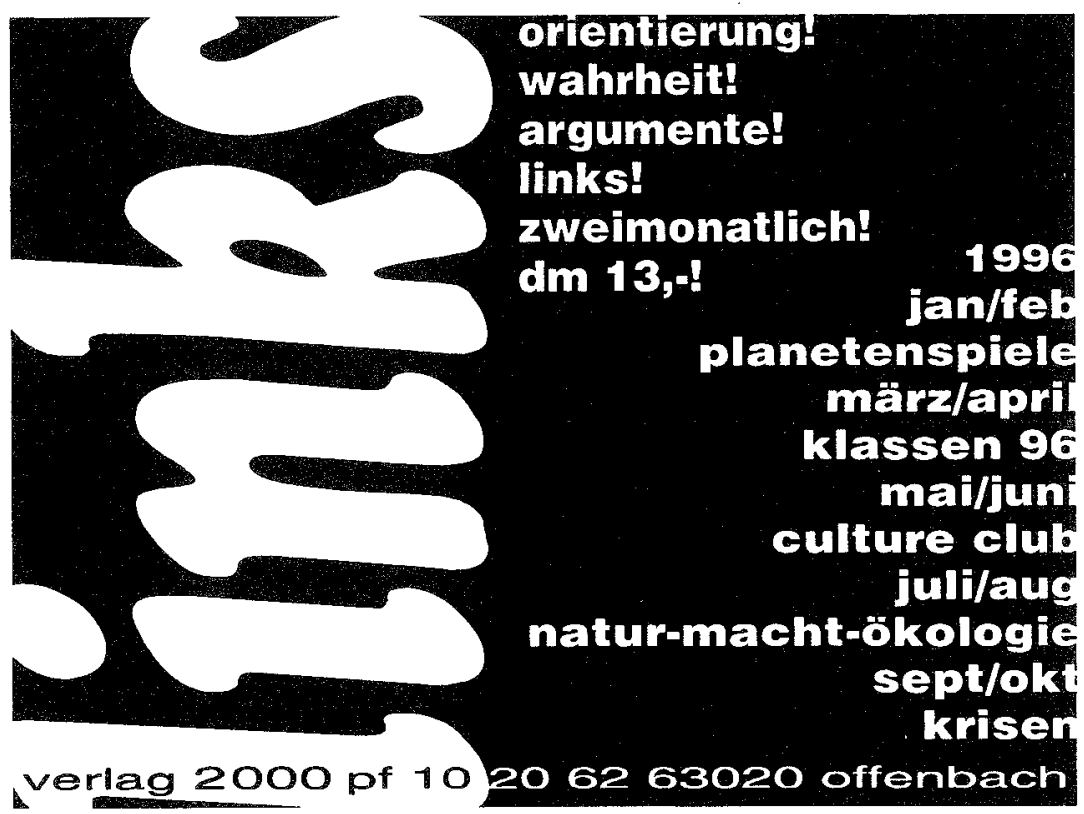

